\title{
Field dependence of the vortex core size in a multi-band superconductor
}

\author{
F.D. Callaghan ${ }^{1}$, M. Laulajainen ${ }^{1}$, C.V. Kaiser ${ }^{1}$, and J.E. Sonier ${ }^{1,2}$ \\ ${ }^{1}$ Department of Physics, Simon Fraser University, Burnaby, British Columbia V5A 1S6, Canada \\ ${ }^{2}$ Canadian Institute for Advanced Research, 180 Dundas Street West, Toronto, Ontario M5G 1Z8, Canada
}

(Dated: August 1, 2021)

\begin{abstract}
The magnetic field dependence of the vortex core size in the multi-band superconductor $\mathrm{NbSe}_{2}$ has been determined from muon spin rotation measurements. The spatially extended nature of the quasiparticle core states associated with the smaller gap leads to a rapid field-induced shrinkage of the core size at low fields, while the more tightly bound nature of the states associated with the larger gap leads to a field-independent core size for fields greater than $4 \mathrm{kOe}$. A simple model is proposed for the density of delocalized core states that establishes a direct relationship between the field-induced reduction of the vortex core size and the corresponding enhancement of the electronic thermal conductivity. We show that this model accurately describes both $\mathrm{NbSe}_{2}$ and the single-band superconductor $\mathrm{V}_{3} \mathrm{Si}$.

PACS numbers: 74.25.Ha, 74.25.Jb, 74.25.Qt, 74.70.Ad, 76.75.+i
\end{abstract}

The discovery of $\mathrm{MgB}_{2}$ [1] , a seemingly conventional type-II superconductor with a high transition temperature $T_{c}=39 \mathrm{~K}$, has sparked considerable experimental and theoretical interest in the physics of multi-band superconductivity (MBSC). MBSC, which was first treated theoretically in 1959 using BCS theory [2], implies that below $T_{c}$ distinct energy gaps open up on different sheets of the Fermi surface. In $\mathrm{MgB}_{2}$, a second smaller gap is induced on the $\pi$ bands through coupling to the intrinsically superconducting $\sigma$ bands. While the high value of $T_{c}$ and the large boron isotope effect in $\mathrm{MgB}_{2}$ are now believed to originate from strong electron-phonon coupling on the two-dimensional $\sigma$ bands, it is also clear that several properties of $\mathrm{MgB}_{2}$ are a manifestation of MBSC 3, 4, 5, 6, 7]. Large vortex cores have been observed on the $\pi$ bands by scanning tunneling spectroscopy [3]. Microscopic calculations of the local density of states (DOS) in a two band superconductor predict that the smaller gap will give rise to spatially extended quasiparticle (QP) states at low field [8, 9]. At higher field, these loosely bound states become delocalized, with the core size now being determined by the more localized states associated with the larger gap. This model is supported by the magnetic field dependence of the electronic thermal conductivity [10, 11] and the specific heat [12, 13, 14], techniques which are sensitive to the delocalization of QPs.

Muon spin rotation $(\mu \mathrm{SR})$ has proven to be an accurate method for determining the field dependence of the vortex core size [15], however single crystals are required for this kind of measurement. Although there have been several studies of $\mathrm{MgB}_{2}$ in the vortex state by $\mu \mathrm{SR}$ [16, 17, 18, 19, 20], these have been done on powdered samples because of the difficulty of growing large, clean, $\mathrm{MgB}_{2}$ single crystals.

$\mathrm{NbSe}_{2}$ has long been thought of as a typical $s$-wave type-II superconductor. However, there is now convincing evidence that it too is a multi-band superconductor [21, 22, 23, 24]. Experiments suggest that an energy gap of magnitude $\sim 1 \mathrm{meV}$ exists on two bands derived from $\mathrm{Nb} 4 d$ orbitals, and a gap $\sim 2-3$ times smaller also exists on a band derived from Se $4 p$ orbitals. In the quest to fully understand the physics of MBSC, it is important to study superconductors other than $\mathrm{MgB}_{2}$, and to this end we performed $\mu \mathrm{SR}$ measurements of the field dependence of the vortex core size in $\mathrm{NbSe}_{2}$ at very low temperature. A distinct advantage of $\mathrm{NbSe}_{2}$ is the availability of large, high quality, single crystals. Single crystals allow for the alignment of the initial muon spin polarization along a single principal crystallographic axis. In this case the $\mu \mathrm{SR}$ lineshape resembles the asymmetric theoretical internal magnetic field distribution $n(B)$ of the vortex lattice. In particular, the high-field tail is the contribution of the vortex cores to $n(B)$ (see Fig. 1) 25]. Previous $\mu \mathrm{SR}$ measurements of the field-dependence of the core size in $\mathrm{NbSe}_{2}$ were performed at $T \geq 2.3 \mathrm{~K}[26]$. At such temperatures, thermal excitations of the bound core states (the so-called 'Kramer-Pesch effect' 27, 28]) result in significant overlap of the QP wavefunctions of adjacent vortices. When this happens the QPs delocalize, giving rise to a strongly field-dependent core size [29], as predicted by the microscopic theory [30].

In order to freeze out thermal excitations of the bound core states and fully isolate the effect of magnetic field on the core size, we have carried out $\mu \mathrm{SR}$ measurements on a single crystal of $\mathrm{NbSe}_{2}\left(T_{c}=7.0 \mathrm{~K}\right.$ and upper critical field $H_{c 2}=45 \mathrm{kOe}$ ) in a dilution refrigerator at $T=20 \mathrm{mK}$. This also permits a direct comparison with electronic thermal conductivity $\kappa_{e}$ data, which is a measure of the extent of QP delocalization. The experiment was performed at the Tri-University Meson Facility (TRIUMF), Vancouver, Canada, with the magnetic field applied parallel to the crystallographic [001] axis. For each measurement, the field was applied at $T>T_{c}$ before cooling the sample to the desired temperature.

The spin-polarized positive muons implanted into the sample stop randomly on the length scale of the vortex lattice, and therefore uniformly sample $n(B)$. Each muon precesses around the local field $B$ at its site at the Larmor frequency $\omega=\gamma_{\mu} B$, where $\gamma_{\mu} / 2 \pi=135.5342$ $\mathrm{MHz} / \mathrm{T}$ is the muon gyromagnetic ratio. On decay of 


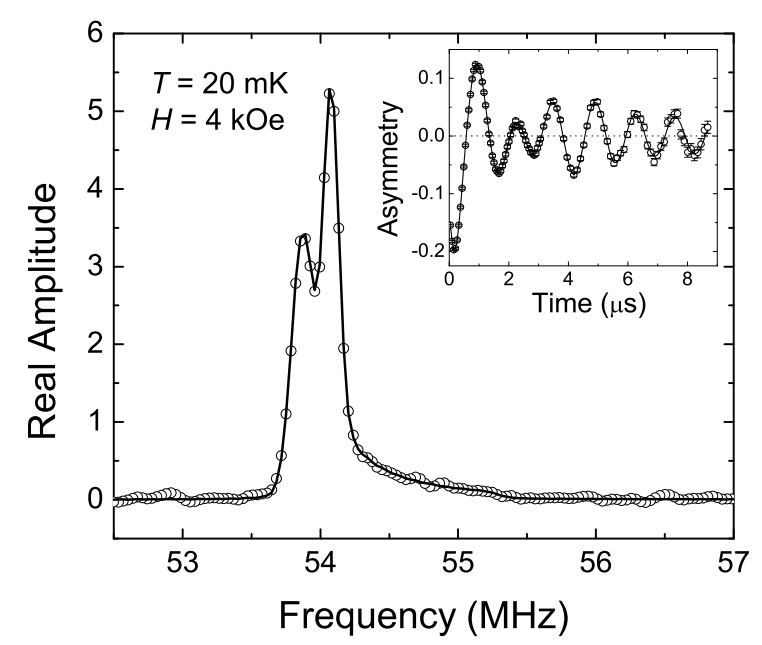

FIG. 1: Fast Fourier transforms of both the time dependence of the muon spin polarization in $\mathrm{NbSe}_{2}$ at $T=20 \mathrm{mK}$ and $H=4 \mathrm{kOe}$ (open circles) and the fit (solid curve) to Eq. (1). Inset: muon spin precession signal and fit to Eq. (1) (in a reference frame rotating at $53.3 \mathrm{MHz}$ ).

the muon after an average lifetime of $2.2 \mu \mathrm{s}$, a positron is emitted preferentially along the direction of the muon spin. The time evolution of the muon spin polarization is determined by detecting decay positrons from an ensemble of $\sim 2 \times 10^{7}$ muons. The exact functional form of the muon spin polarization depends on $n(B)$. Further details of the experimental technique used here can be found in Ref. [25]. The $\mu \mathrm{SR}$ time spectra were fit assuming a Ginzburg-Landau (GL) model for the spatial field profile given by

$$
B(\mathbf{r})=B_{0}\left(1-b^{4}\right) \sum_{\mathbf{G}} \frac{e^{-i \mathbf{G} \cdot \mathbf{r}} u K_{1}(u)}{\lambda_{a b}^{2} G^{2}}
$$

where $b=B / B_{c 2}, B_{0}$ is the average internal field, $\mathbf{G}$ are the reciprocal lattice vectors, $K_{1}(u)$ is a modified Bessel function, $u^{2}=2 \xi_{a b}^{2} G^{2}\left(1+b^{4}\right)\left[1-2 b(1-b)^{2}\right], \xi_{a b}$ is the GL coherence length, and $\lambda_{a b}$ is the magnetic penetration depth. As explained in Ref. 15], previous $\mu \mathrm{SR}$ works have demonstrated that the field-dependence of the parameter $\xi_{a b}$ reflects changes in the vortex core size due to changes in the electronic structure of the vortex cores.

Figure 1 shows fast Fourier transforms (FFTs) of both the muon spin precession signal at $H=4 \mathrm{kOe}$ and the fit to Eq. (1). The FFTs closely resemble $n(B)$ for a hexagonal vortex lattice, but are significantly broadened by the apodization used to reduce ringing and noise in the FFT due to the finite time-range and the short muon lifetime, respectively. The lineshape is also broadened by a Gaussian distribution of fields from randomly oriented nuclear dipolar moments. The width of this distribution

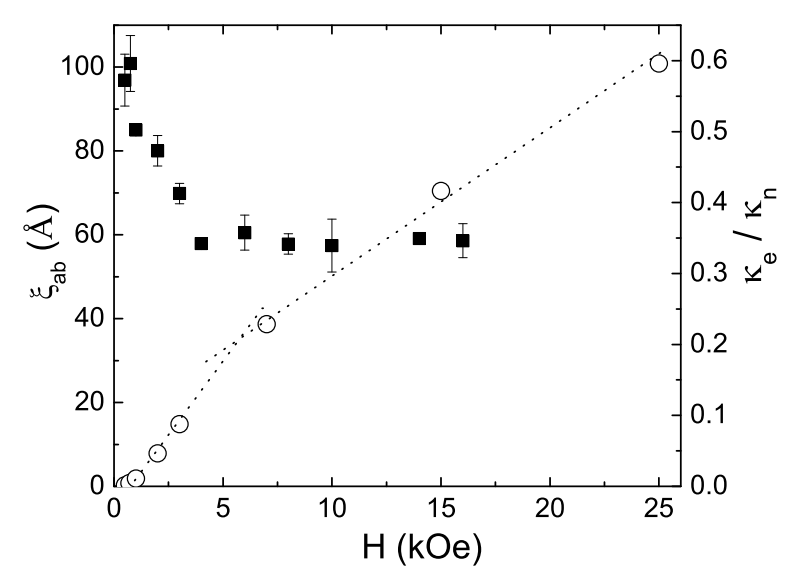

FIG. 2: Field dependence of the vortex core size $\xi_{a b}$ at $T=$ $20 \mathrm{mK}$ (squares) and the electronic thermal conductivity $\kappa_{e}$ normalised to its normal-state value $\kappa_{n}$ (circles). The dashed lines are guides for the eye.

was determined to be $\approx 0.25 \mathrm{MHz}$. In addition, there is a background peak at $\sim 54.1 \mathrm{MHz}$ originating from muons stopping outside the sample.

Recently, $\mu \mathrm{SR}$ measurements on powder samples of $\mathrm{Mg}_{1-x} \mathrm{Al}_{x} \mathrm{~B}_{2}$ were modeled assuming two distinct energy gaps, and a two-component field distribution for the vortex lattice [19]. Specifically, this model assumes two distinct coherence lengths, which implies that both large and small vortex cores exist simultaneously in the sample. However, for $\mathrm{NbSe}_{2}$ we find that the data are well described by Eq. (11). A visual inspection of Fig. 1 shows that the fit captures all of the main features of the $\mu \mathrm{SR}$ lineshape. This is consistent with recent theoretical work predicting that the formation of two vortex sublattices is energetically forbidden in a two-gap superconductor [31].

Recent $\mu \mathrm{SR}$ measurements of the field dependence of the core size in the single-band superconductor $\mathrm{V}_{3} \mathrm{Si}$ [29] have confirmed theoretical predictions of field-induced core shrinkage due to QP delocalization [30]. In the case of $\mathrm{NbSe}_{2}$, the delocalization of core states is dependent on both energy gaps. At low fields the rapid reduction of $\xi_{a b}$ and the simultaneous rapid increase of $\kappa_{e}$ with increasing magnetic field are consistent with this regime being dominated by loosely bound states associated with the smaller energy gap [8, 9]. If the loosely bound core states associated with the smaller gap have completely delocalized by $H \approx 4 \mathrm{kOe}$, then we expect the behavior of $\xi_{a b}$ at larger fields to be dominated by the larger gap. The saturation of $\xi_{a b}$ above $4 \mathrm{kOe}$ indicates that the QPs are highly localized in the vortex cores at higher fields. This is in line with STM measurements which show that the bound core states at $H=10 \mathrm{kOe}$ are more spatially confined than those at $H=1 \mathrm{kOe}[32]$. We note that even though the shrinking of the core size saturates 


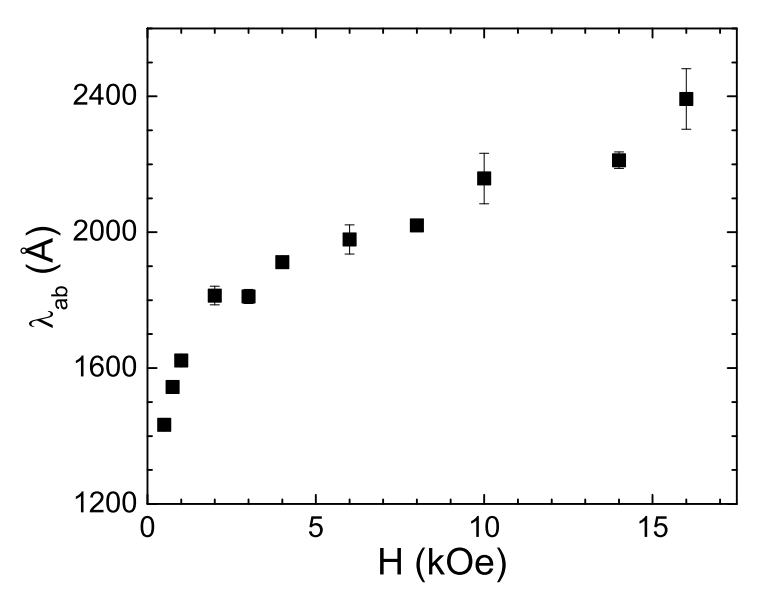

FIG. 3: Field dependence of the magnetic penetration depth in $\mathrm{NbSe}_{2}$ at $T=20 \mathrm{mK}$.

above $4 \mathrm{kOe}, \kappa_{e}$ still exhibits a considerable dependence on field. The reason is that although the number of delocalized QPs per vortex is no longer changing, the increasing number of vortices increases the total number of delocalized QPs (i.e. heat carriers) in the sample.

Given that the core size at low (high) field is primarily determined by the small (large) gap, one might naively expect that the BCS relationship $\xi \sim v_{F} / \pi \Delta_{0}$, where $v_{F}$ is the Fermi velocity and $\Delta_{0}$ is the magnititude of the energy gap, could be used to determine the ratio of the high and low field core sizes. However, recent calculations based on GL theory have shown that this relationship is not applicable to a multi-band superconductor and that a simple expression for the ratio of the two core sizes only exists in the limit of zero interband coupling 33]. For finite interband coupling, the ratio must be calculated numerically and is dependent on a number of material parameters.

As can be seen from Eq. (1), the magnetic penetration depth $\lambda_{a b}$ is also a fitting parameter in our analysis, extracted simultaneously with $\xi_{a b}$. Fig. 3 shows that $\lambda_{a b}$ increases strongly with $H$ at low fields and displays a weaker, though significant, dependence at higher fields. The Volovik effect [34] has previously been invoked to explain the field dependence of $\lambda_{a b}$ in $s$-wave type-II superconductors [35]. This effect involves a shift of the quasiparticle energy spectrum by an energy $\sim \mathbf{v}_{\mathbf{F}} \cdot \mathbf{v}_{\mathbf{S}}$, where $\mathbf{v}_{\mathbf{S}}$ is the supercurrent velocity, and it plays a significant role in superconductors with gap nodes. However, for an isotropic $s$-wave energy gap, a significant dependence of $\lambda_{a b}$ on $H$ is only expected if the thermal energy $k_{B} T$ (where $k_{B}$ is Boltzmann's constant) is comparable to the magnitude of the energy gap. At $T=20 \mathrm{mK}$, $k_{B} T \approx 10^{-3} \mathrm{meV}$, whereas the size of the small energy gap in $\mathrm{NbSe}_{2}$ is $\approx 10^{-1} \mathrm{meV}$. Therefore we cannot attribute the observed behaviour of $\lambda(H)$ to the Volovik effect.

Calculations of the electronic structure of a single vortex using the Bogoliubov-deGennes equations have shown that the presence of extended quasiparticle states significantly modifies the current density, and hence $B(\mathbf{r})$, around the vortex core [36]. With increasing magnetic field these modified regions overlap. Since Eq. (1) does not account for this, the effect on $B(\mathbf{r})$ shows up in our measurements as a field dependent $\lambda_{a b}$. In other words $\lambda_{a b}$ in Eq. (1) is an effective magnetic penetration depth. We note that the field dependence of $\lambda_{a b}$ is stronger at low field due to the more rapid delocalization of QPs from the vortex cores.

Next we propose a simple model which directly relates our measurements of the vortex core size to the measurements of the electronic thermal conductivity. In the conventional picture of a type-II superconductor, the density of localized QP states at the Fermi energy $N\left(E_{F}\right)_{\text {loc }}$ is proportional to $\pi \xi^{2} H$, where $\pi \xi^{2}$ is the area of a single vortex core and the density of vortices in the sample is proportional to $H$ 37, 38]. If we assume that the fieldinduced density of delocalized QP states is equal to the reduction in $N\left(E_{F}\right)_{\text {loc }}$, then

$$
N\left(E_{F}\right)_{\text {deloc }} \propto\left(\pi \xi_{0}^{2}-\pi \xi^{2}\right) H
$$

where $\xi_{0} \approx 97 \AA$ is the low-field value of the core size. Note that $\left(\pi \xi_{0}^{2}-\pi \xi^{2}\right) H$ is also proportional to the reduction of the total core area in the sample due to the delocalization of QP core states.

In a metal, $\kappa_{e}=(1 / 3) C v_{F} l=(1 / 9) \pi^{2} N\left(E_{F}\right) v_{F} l k_{B}^{2} T$, where $C$ is the heat capacity per unit volume, $k_{B}$ is Boltzmann's constant, $v_{F}$ is the Fermi velocity, and $l$ is the electron mean free path [39]. Since it is the delocalized QPs that carry heat then $\kappa_{e} \propto N\left(E_{F}\right)_{\text {deloc }}$, and it follows from Eq. (2) that

$$
\kappa_{e} \propto\left(\pi \xi_{0}^{2}-\pi \xi^{2}\right) H
$$

To show that this relationship is physically valid, in Fig. 4 we plot the measured quantities $\kappa_{e}$ and $\left(\pi \xi_{0}^{2}-\pi \xi^{2}\right) H$ against $\left(H_{c 2}(0) / H\right)^{1 / 2}$, which is proprtional to the intervortex spacing. We also plot our earlier measurements on $\mathrm{V}_{3} \mathrm{Si}$ (Ref. [29]) in this way. It can be seen that at large intervortex spacing (low $H$ ) neither quantity changes significantly. However, as the field is increased and the vortices are brought closer together, both quantities exhibit the same field dependence within experimental uncertainty. This lends further support to the conclusion that in both single-band $\mathrm{V}_{3} \mathrm{Si}$ and multiband $\mathrm{NbSe}_{2}$ the field dependences of $\xi_{a b}$ and $\kappa_{e}$ have a common underlying cause, namely the delocalization of bound QPs.

In summary, we have measured the field dependence of the vortex core size in the multi-band superconductor $\mathrm{NbSe}_{2}$ at $T=20 \mathrm{mK}$. The observed field dependence is explained by the effects of two superconducting energy gaps on the bound core states. In addition, we have experimentally established a direct correlation between 


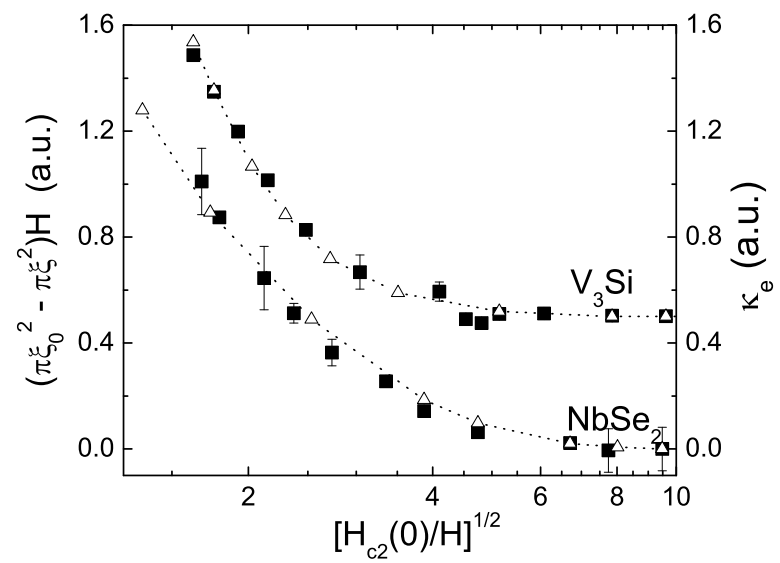

FIG. 4: Total reduction in core area (left axis, solid squares) and electronic thermal conductivity $\kappa_{e}$ (right axis, open triangles) plotted against $\left(H_{c 2} / H\right)^{1 / 2}$ (which is proportional to the intervortex spacing) for $\mathrm{V}_{3} \mathrm{Si}$ and $\mathrm{NbSe}_{2}$. Both quantities are normalised to their values at $H \sim 70 \mathrm{kOe}$ for $\mathrm{V}_{3} \mathrm{Si}$ and $H \sim 10 \mathrm{kOe}$ for $\mathrm{NbSe}_{2}$. The dashed lines connect the $\kappa_{e}$ data points. (The $\mathrm{V}_{3} \mathrm{Si}$ data are shifted upwards by 0.5 on the vertical scale for clarity.) the field-induced reduction of the vortex core area and the electronic thermal conductivity in both single-band and multi-band superconductors.
This work was supported by the Natural Sciences and Engineering Research Council (NSERC) of Canada and the Canadian Institute for Advanced Research (CIAR). The authors wish to thank the TRIUMF support staff for technical assistance, Roger Miller for useful discussions and assistance with data acquisition, and J.W. Brill for providing the $\mathrm{NbSe}_{2}$ sample.
[1] J. Nagamatsu, N. Nakagawa, T. Muranaka, Y. Zenitani, and J. Akimitsu, Nature 410, 63 (2001).

[2] H. Suhl, B.T. Matthias, and L.R. Walker, Phys. Rev. Lett. 3, 552 (1959).

[3] M.R. Eskildsen et al., Phys. Rev. Lett. 89, 187003 (2002).

[4] R.S. Gonnelli et al., Phys. Rev. Lett. 89, 247004 (2002).

[5] R. Cubitt et al., Phys. Rev. Lett. 91, 047002 (2003).

[6] S. Tsuda et al., Phys. Rev. Lett. 91, 127001 (2003).

[7] Y. Bugoslavsky et al., Phys. Rev. B 69, 132508 (2004).

[8] N. Nakai, M. Ichioka, and K. Machida, J. Phys. Soc. Jpn. 71, 23 (2002).

[9] M. Ichioka, K. Machida, N. Nakai, and P. Miranović, Phys. Rev. B 70, 144508 (2004).

[10] A.V. Sologubenko, J. Jun, S.M. Kazakov, J. Karpinski, and H.R. Ott, Phys. Rev. B 66, 014504 (2002).

[11] H. Kusunose, T.M. Rice, and M. Sigrist, Phys. Rev. B 66, 214503 (2002).

[12] H.D. Yang et al., Phys. Rev. Lett. 87, 167003 (2001).

[13] F. Bouquet et al., Phys. Rev. Lett. 89, 257001 (2002).

[14] L. Tewordt and D. Fay, Phys. Rev. B 67, 134524 (2003).

[15] J.E. Sonier, J. Phys. Condens. Matter 16, S4499 (2004).

[16] C. Panagopoulos et al., Phys. Rev. B 64, 094514 (2001).

[17] Ch. Niedermayer, C. Bernhard, T. Holden, R.K. Kremer, and K. Ahn, Phys. Rev. B 65, 094512 (2002).

[18] K. Ohishi et al., J. Phys. Soc. Jpn. 72, 29 (2003).

[19] S. Serventi et al., Phys. Rev. Lett. 93, 217003 (2004).

[20] M. Angst et al., Phys. Rev. B 70, 224513 (2004).

[21] R. Corcoran et al., J. Phys. Condens. Matter 6, 4479
(1994).

[22] T. Yokoya et al., Science 294, 2518 (2001).

[23] E. Boaknin et al., Phys. Rev. Lett. 90, 117003 (2003).

[24] J.G. Rodrigo and S. Vieira, Physica C 404, 306 (2004).

[25] J.E. Sonier, J.H. Brewer, and R.F. Kiefl, Rev. Mod. Phys. 72, 769 (2000).

[26] J.E. Sonier et al., Phys. Rev. Lett. 79, 1742 (1997).

[27] L. Kramer and W. Pesch, Z. Phys. 269, 59 (1974).

[28] R.I. Miller et al., Phys. Rev. Lett. 85, 1540 (2000).

[29] J.E. Sonier et al., Phys. Rev. Lett. 93, 017002 (2004).

[30] M. Ichioka, A. Hasegawa, and K. Machida, Phys. Rev. B 59, 184 (1999).

[31] E. Babaev, Phys. Rev. Lett. 89, 067001 (2002).

[32] H.F. Hess, R.B. Robinson, R.C. Dynes, J.M. Valles, Jr., and J.V. Waszczak, J. Vac Sci. Technol. A 8, 450 (1990).

[33] M.E. Zhitomirsky and V.-H. Dao, Phys. Rev. B 69, 054508 (2004).

[34] G.E. Volovik, JETP Lett. 58, 457 (1993).

[35] R. Kadono, J. Phys. Condens. Matter 16, S4421 (2004).

[36] F. Gygi and M. Schlüter, Phys. Rev. B 43, 7609 (1991).

[37] C. Caroli, P.G. de Gennes, and J. Matricon, Phys. Lett. 9, 307 (1964).

[38] A.L. Fetter and P. Hohenberg in Superconductivity, edited by R.D. Parks (Marcel Dekker Inc., New York, 1969), Vol. 2, pp. 817-923.

[39] Charles Kittel, Introduction to Solid State Physics, 7th ed. (Wiley, 1996). 